\section{JTI}

JOURNAL OF

TRAUMA AND INJURY

Received: March 16, 2020

Revised: June 3, 2020

Accepted: June 4, 2020

\section{Correspondence to}

Chang-Wug Oh, M.D., Ph.D.

Department of Orthopedic Surgery, Kyungpook National University Hospital, School of Medicine, Kyungpook National University, 130 Dongdeok-ro, Jung-gu,

Daegu 41944, Korea

Tel: $+82-53-420-5630$

Fax: +82-53-422-6605

E-mail:cwoh@knu.ac.kr

\title{
Exchange Nailing for Aseptic Nonunion of the Femoral Shaft after Intramedullary Nailing
}

\author{
Sung-Soo Ha, M.D. ${ }^{1,2}$, Chang-Wug Oh, M.D., Ph.D. ${ }^{1,2}$, Jae-Wook Jung, M.D. ${ }^{1,2}$, \\ Joon-Woo Kim, M.D., Ph.D. ${ }^{1,2}$, Kyeong-Hyeon Park, M.D., Ph.D. ${ }^{1,2}$, \\ Seong-Min Kim, M.D., \\ ${ }^{1}$ Regional Trauma Center, Kyungpook National University Hospital, Daegu, Korea \\ ${ }^{2}$ Department of Orthopaedic Surgery, School of Medicine, Kyungpook National University, \\ Daegu, Korea
}

Purpose: Although exchange nailing is a standard method of treating femoral shaft nonunion, various rates of healing, ranging from $72 \%$ to $100 \%$, have been reported. The purpose of this study was to evaluate the efficacy of exchange nailing in femoral shaft nonunion.

Methods: We retrospectively reviewed 30 cases of aseptic femoral shaft nonunion after intramedullary nailing. The mean postsurgical period of nonunion was 66.8 weeks. A nail at least $2 \mathrm{~mm}$ larger in diameter was selected to replace the previous nail after reaming. Distal fixation was performed using at least two interlocking screws. The success of the procedure was determined by the finding of union on simple radiographs. Possible reasons for failure were analyzed, including the location of nonunion, the type of nonunion, and the number of screws used for distal fixation.

Results: Of the 30 cases, 27 achieved primary healing with the technique of exchange nailing. The average time to achieve union was 23.1 weeks (range, 13.7-36.9 weeks). The three failures involved nonunion at the isthmic level (three of 15 cases), not at the infraisthmic level (zero of 15 cases). Of eight cases of oligotrophic nonunion, two (25\%) failed to heal, and of 22 cases of hypertrophic nonunion, one (4.5\%) failed to heal. Of 11 cases involving two screws at the distal fixation, two (18.2\%) failed to heal, and of 19 cases involving three or more screws, one (5.3\%) failed to heal. None of these findings was statistically significant.

Conclusions: Exchange nailing may enable successful healing in cases of aseptic nonunion of the femoral shaft. Although nonunion at the isthmic level, oligotrophic nonunion, and weaker distal fixation seemed to be associated with a higher chance of failure, further study is needed to confirm those findings.

Keywords: Femoral shaft nonunion; Exchange nailing; Risk factors; Distal interlocking 


\section{INTRODUCTION}

Intramedullary nailing is the treatment of choice for femoral shaft fractures in adults; however, relatively high rates of nonunion have been reported $(4.1-12 \%)$ [1,2]. The treatment options for femoral shaft nonunion include dynamization, bone graft, exchange nailing, and augmentation plating without nail removal $[3,4]$. In addition, biological adjuvant therapy-including extracorporeal shock wave therapy and the use of recombinant bone morphogenic protein-7, platelet-rich plasma, reamerirrigator-aspirator grafts, and bone marrow aspirate-have been reported. Exchange nailing is the standard operative technique for femoral shaft nonunion, and it does not require opening of or resection at the nonunion site $[5,6]$. The advantages of this technique include minimal blood loss, low rates of surgical morbidity, and no need for a bone graft because the fracture site is not exposed.

Various rates of successful healing with the exchange nailing procedure have been reported $[7,8]$. Although high success rates were reported initially, recent studies have revealed less encouraging results, with the requirement of additional surgical interventions. Several factors have been reported to be associated with failure, including initial open fracture, low-grade infections, associated injury, the type of nonunion, and the location of nonunion. In many of the previous reports, patient demographics have been heterogeneous, even among cases of infected nonunion $[5,6,8]$. Furthermore, given the variety of methods of primary treatment, analyzing the success or failure of exchange nailing may be difficult. The literature still lacks a clear consensus about the indications, risk factors for failure, and limitations of femoral exchange nailing.

In this study, we collected data from cases of nonunion that developed after intramedullary nailing was used as the primary method of treatment for femoral shaft fracture. Excluding cases of infected nonunion, we assessed the outcomes of cases treated by exchange nailing. We hypothesized that the success of exchange nailing could be affected by the location of nonunion, the type of nonunion, and/or the number of screws used in distal fixation. The aim of this study was to report the results of exchange nailing in the treatment of femoral shaft nonunion.

\section{METHODS}

\section{Patients and methods}

Patients who underwent exchange nailing for the management of femoral shaft nonunion after intramedullary nailing between March 2006 and April 2016 were included. A retrospective analysis was performed on data from 30 patients who were available for follow-up for at least 12 months. All fractures were classified according to the AO Foundation/Orthopaedic Trauma Association (AO/ OTA) classification. Fracture nonunion was defined as no signs of union at 6 months after primary surgery with no evidence of progression of healing in the previous 3 months. Cases were classified as hypertrophic or oligotrophic nonunion on the basis of findings on simple radiographs.

While the patient was in a supine or lateral position on a radiolucent table, exchange nailing was performed with the help of fluoroscopy. After removal of previous implants, over-reaming of the medullary canal was performed until the fit between the reamer head and the medullary canal was tight. A nail at least $2 \mathrm{~mm}$ in diameter was selected for antegrade insertion. The length and kind of nail-cannulated femoral nail (ITS Implants for Trauma Surgery, Graz, Austria), Expert A2FN (DePuy Synthes, Oberdorf, Switzerland), T2 femoral nail (Stryker Corporation, Kalamazoo, MI, USA), Sirus intramedullary femoral nail (Zimmer Biomet, Warsaw, IN, USA), or Zimmer natural nail (Zimmer Biomet)-were chosen to avoid overlap with the previous holes of interlocking screws, so as to increase the fixation stiffness. In selected cases, Poller screws were added to enhance the stability of the distal segment, and additional interlocking screws were fixed for the same purpose. After fixation of the distal interlocking screws, the back-strike technique was used to minimize the gap of nonunion. We then performed proximal interlocking without surgical exposure of the nonunion site or the bone graft. All patients were allowed to start joint movement immediately after surgery, and weight-bearing was encouraged to the extent tolerated by the patients.

Union was defined as the presence of a bridging callus on at least three cortices, as observed on standard anteroposterior and lateral radiographs. Time to union was 
determined from the day of initial exchange nailing to the date of union according to previously defined radiographic criteria. Nonunion was classified as either hypertrophic or atrophic, according to the criteria proposed by Weber and Brunner [9]. Isthmic nonunion of the femoral shaft was defined as a fracture at the shortest region of the bilateral cortex of the femoral shaft, observed on anteroposterior femur radiographs. Infraisthmic nonunion was defined as a fracture line in the region between the isthmus and the upper border of the transepicondylar width in the knee joint [7].

Statistical analysis (with SPSS software [IBM Corporation, Armonk, NY, USA] and the Fisher's exact test) was performed to identify any factors affecting union after exchange nailing, including the location of nonunion (isthmic versus infraisthmic), nonunion type (hypertrophic versus oligotrophic), and the numbers of screws for distal fixation (two screws versus three or more). Statistical significance was denoted by $p$-values of less than 0.05 .

\section{RESULTS}

Data from 30 patients with femoral shaft nonunion (24 men and six women) were included; the mean age of the patients was 51.3 years (range, 18-78 years). According to the AO/OTA classification, 15 patients had a simple fracture (32-A), 11 had a wedge fracture (32-B), and four had a multifragmentary fracture (32-C). According to the radiographic features, 22 cases were hypertrophic nonunion and eight were oligotrophic nonunion. According to the level of nonunion at the index operation, 15 fractures were isthmic and 15 were infraisthmic.

The mean time between primary fixation and exchange nailing was 66.8 weeks (range, 18.6-200 weeks). Various kinds of nails (12 cannulated femoral nails, nine expert A2FN, six T2 femoral nails, one Sirus intramedullary femoral nail, and two Zimmer natural nails) were used to fix interlocking screws to the new holes to achieve better stability. Different numbers of nails were used in distal fixation: two interlocking screws were used in 11 cases (10 isthmic fractures and one infraisthmic fracture) and three or more interlocking screws (including Poller screws) were used in 19 cases (five isthmic fractures and 14 in- fraisthmic fractures). After exchange nailing, union was achieved in 27 patients (90\%) in an average of 23.1 weeks (range, 13.7-36.9 weeks). In three patients, nonunion persisted.

In all three cases of nonunion, the failures were at the isthmic level of the femoral shaft; none were at the infraisthmic level ( $p>0.05$, Fisher's exact test). With regard to the classification of nonunion, two (25\%) of the eight cases of oligotrophic nonunion failed to heal, and one (4.5\%) of the 22 cases of hypertrophic nonunion failed $(p>0.05$, Fisher's exact test). Of the 11 cases in which two screws were used, two (18.2\%) resulted in failure, and of 19 cases involving three or more screws, one (5.3\%) resulted in failure ( $p>0.05$, Fisher's exact test). Thus, the possible factors contributing to failure after exchange nailing were not significantly different among the different types and locations of fractures or according to the number of nails used.

No other complications, including infections, implant breakage (nail or interlocking screw), and malalignment of more than $5^{\circ}$, occurred. All patients were able to perform full weight-bearing activities 3 months after surgery. The range of movement of the knee joint before surgery did not differ significantly from that after surgery. All patients achieved comfortable daily activities, similar to the preinjury level. In three cases of persistent nonunion, additional procedures were needed to achieve union.

\section{Case 1}

A patient sustained a femoral shaft fracture with a medial bending wedge fragment (AO/OTA 32-B2) at the isthmic level, which was repaired with closed intramedullary nailing. Eleven months later, oligotrophic nonunion developed, and the medial wedge fragment was displaced from the main shaft segment. Exchange nailing was performed with a larger diameter nail and two distal interlocking screws. Nonunion was found to have recurred, mainly on the medial side, at the 10-month follow-up. A second exchange nailing was performed with four distal interlocking screws, and union was achieved 12 months later. The displacement of the medial fragment might have limited the contact between the nail and the endosteal surface. Inadequate stability was presumed to be the cause of nonunion after exchange nailing, although the nonunion was 
at the isthmic level (Fig. 1).

\section{Case 2}

A patient experienced a fracture of the ipsilateral femoral neck and shaft (AO/OTA 32-A3) at the isthmic level, which was repaired with fixation of hip screws and retrograde nailing. Although the neck fracture healed, hypertrophic nonunion of the femoral shaft developed 8 months after the initial repair. Exchange nailing was performed with two distal interlocking screws. Seven months later, nonunion was found again, and radiographs showed widening of the femoral canal and loosening of distal screws. Exchange nailing was performed again with a larger diameter nail and without a bone graft, and an additional augmenting plate was affixed to the femur at the same time. Because concomitant nonunion of the ipsilateral calcaneus was present at the time of first exchange nailing, we thought that insufficient weight-bearing activity might have weakened the femoral cortex and resulted in limited contact between surfaces at the isthmic level (Fig. 2).

\section{Case 3}

A patient sustained a fracture of the ipsilateral femoral neck and shaft (AO/OTA 32-C2) with polytrauma, which was treated with fixation of hip screws and retrograde nailing. Oligotrophic nonunion at the isthmic level developed 18 months later, but the other fractures healed. Exchange nailing was performed with two distal interlocking screws. At 30 months later, the nonunion still persisted with callus formation, and the space between the nail and endosteal surface was increased. The nail and interlocking screws were intact, and so supplemental augmentation plating was performed without bone graft. Because these fractures were concomitant with tibia fractures in the initial injury, we thought that insufficient weight-bearing activity might have weakened the femoral cortex and resulted in the limited contact between surfaces (Fig. 3).

\section{DISCUSSION}

No statistically significant relationships were found between nonunion of the femoral shaft and the risk factors
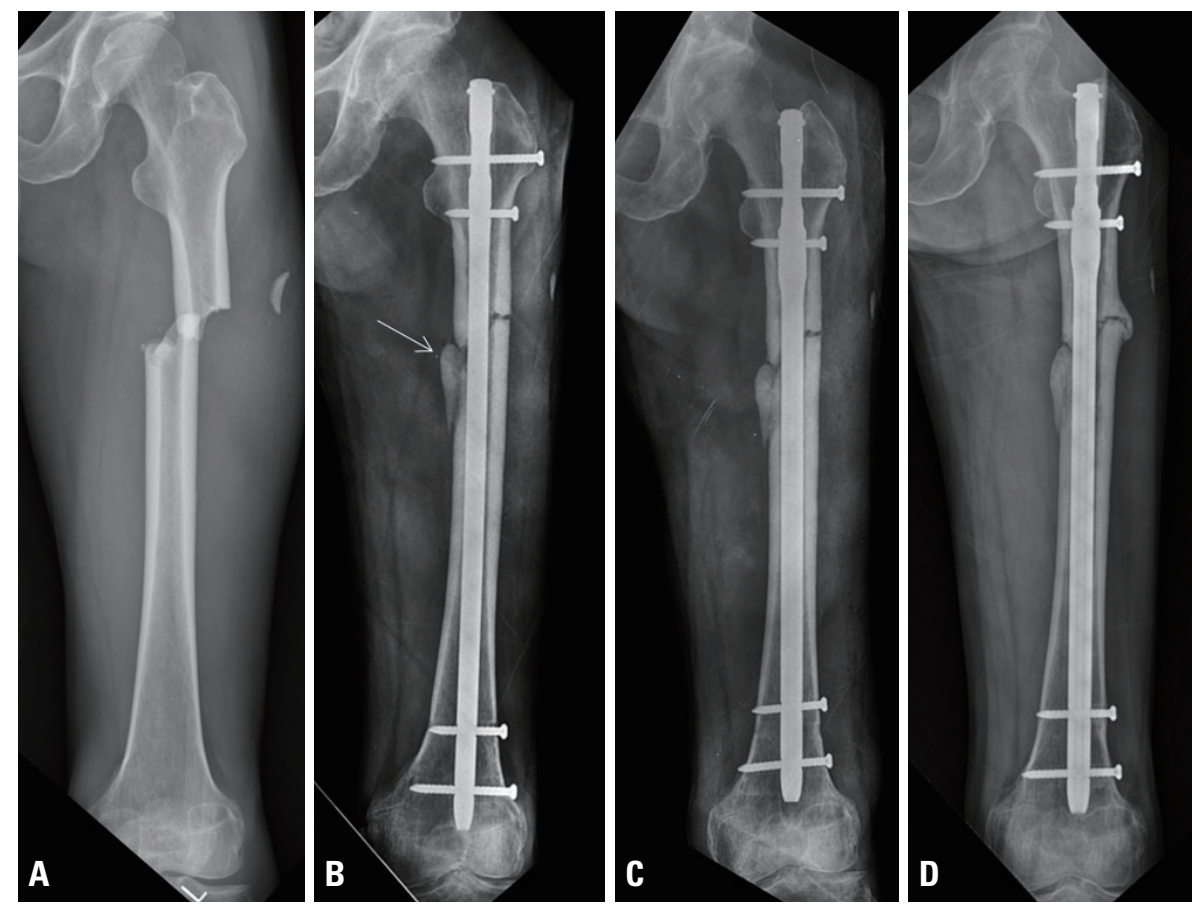

Fig. 1. Case 1. (A) Left femoral shaft fracture at the isthmic level. (B) Oligotrophic nonunion at 11 months with the medial wedge fragment displaced (arrow). (C) Postoperative radiograph after exchange nailing. (D) Nonunion observed again at 10 months after exchange nailing. 

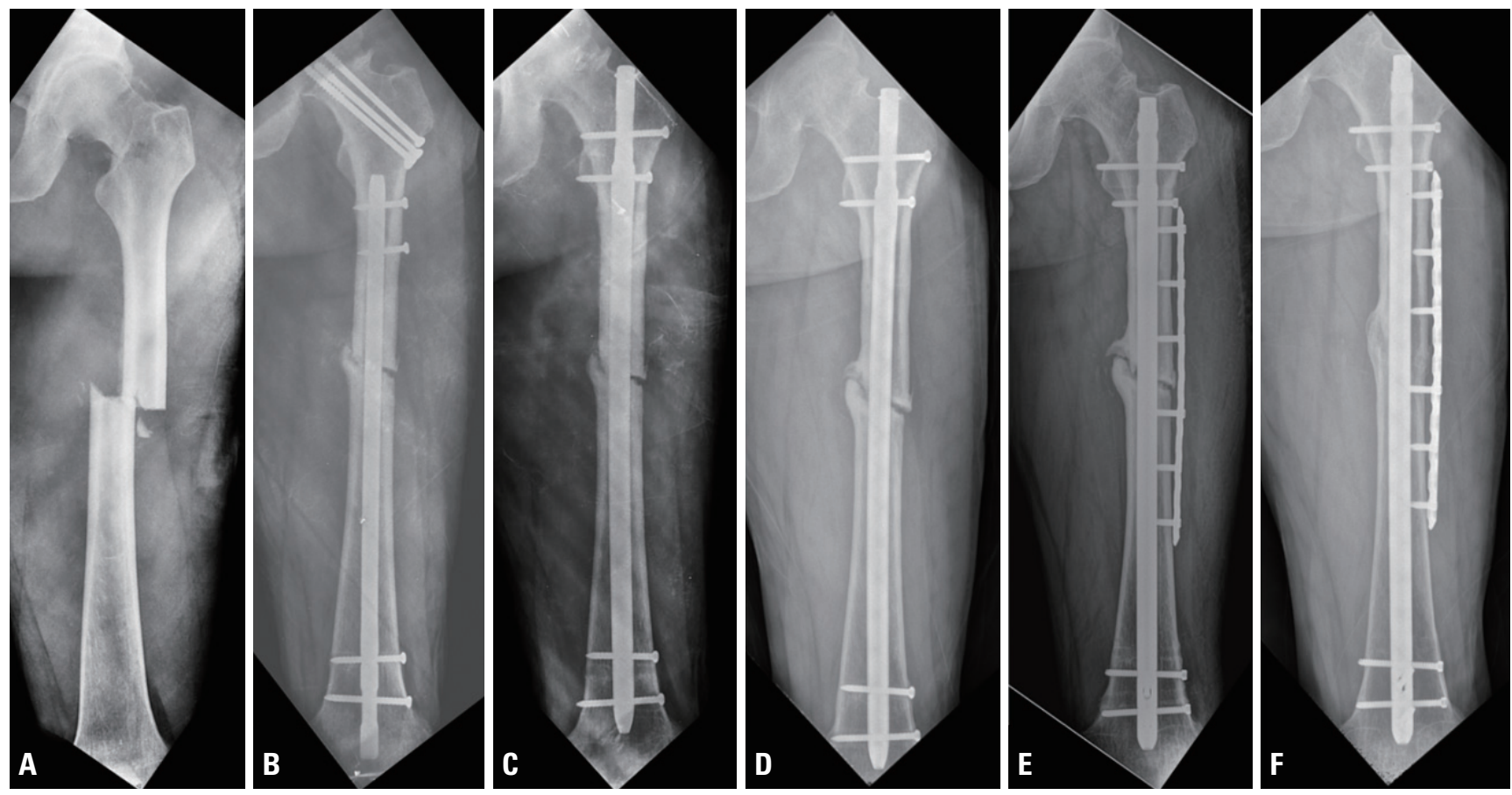

Fig. 2. Case 2. (A) Ipsilateral neck and shaft fracture of the left femur. (B) Hypertrophic nonunion of the isthmic portion at 8 months. (C) Postoperative radiograph after exchange nailing. (D) Nonunion observed again at 7 months after exchange nailing. (E) Second exchange nailing with augmentation plating. (F) United femur at 11 months after the second exchange nailing with augmentation plating.
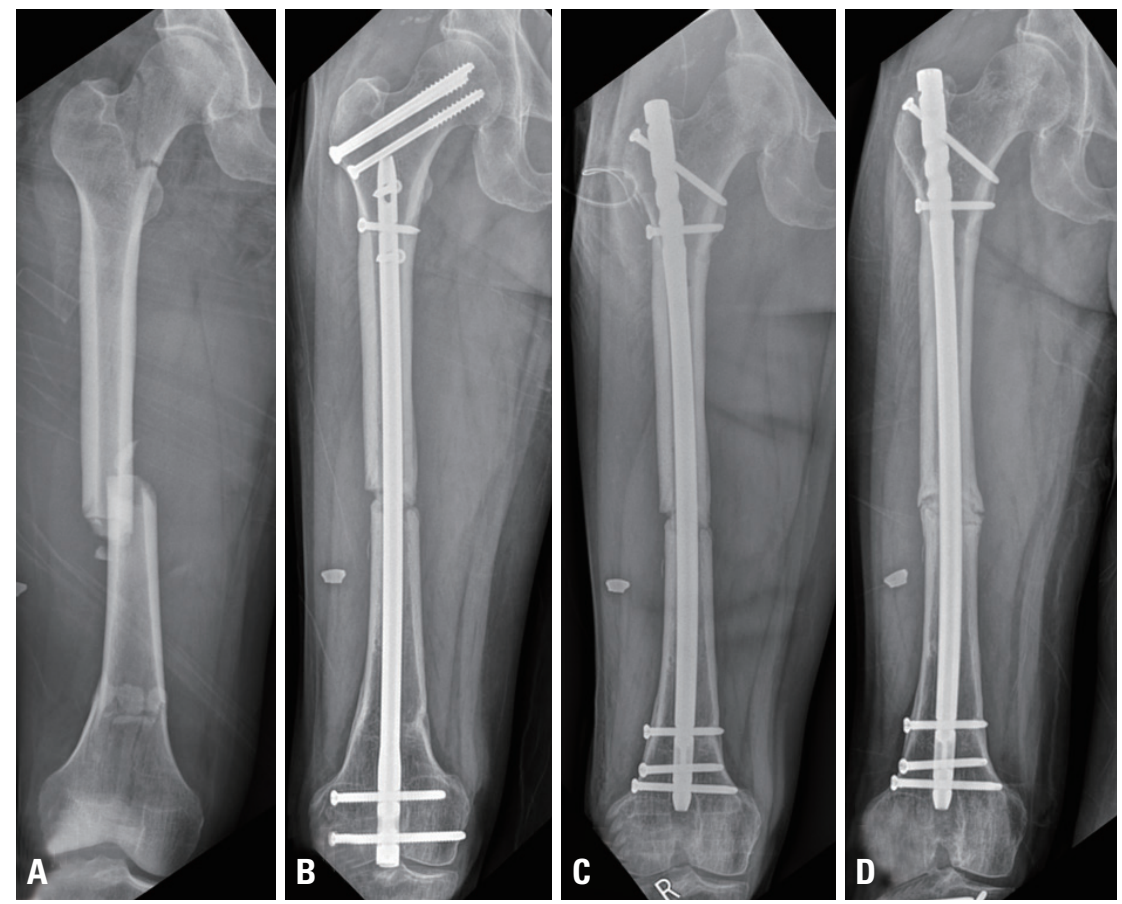

Fig. 3. Case 3. (A) Ipsilateral neck and segmental shaft fracture of the right femur. (B) Oligotrophic nonunion of the isthmic portion at 18 months. (C) Postoperative radiograph after exchange nailing. Nonunion observed again at 30 months after exchange nailing. 
that we expected (location of nonunion, nonunion type, and number of screws used for distal fixation), which may suggest that the exchange nailing without any other additional procedure is a sufficient treatment option for nonunion of the femoral shaft. However, we identified possible risk factors (inadequate stability due to a medial bone defect and limited contact between the fracture surfaces) that might require more stability of the distal fragment. To verify these findings, a large-scale, multicenter study may be needed.

Among the several treatment options for nonunion after nailing of a femoral shaft fracture, exchange nailing is commonly chosen because it is an easy procedure that is associated with decreased pain, and weight-bearing activities can begin soon after surgery $[6,10]$. Exchange nailing results in a combination of increased mechanical stability caused by the insertion of a larger nail and reactivation of biological healing activity caused by the reaming process [5]. In a recent report, a $100 \%$ healing rate was achieved in a series of 50 consecutive patients when exchange nailing was used to treat aseptic femoral nonunion. However, a considerable number of patients $(14[28 \%])$ required a secondary procedure (dynamization). We achieved a comparably high rate of successful healing (90\%) of femoral shaft nonunion by performing exchange nailing with static locking only. However, several factors are known to result in less encouraging outcomes, necessitating additional surgical interventions, including the presence of medical comorbidities or an infection, fracture classification, the location of nonunion, and the type of nonunion $[7,8]$. In particular, low-grade infections, which may be difficult to diagnose before the operation, may contribute to the failure of exchange nailing [11]. Because we excluded cases of infected nonunion, the failure rate (10\%) was low in this study. Patient selection is very important for successful results after exchange nailing [10].

Yang et al. [7] reported that nonisthmal (infraisthmic or supraisthmic) femoral shaft nonunion was a risk factor for failure of exchange nailing, as was isthmic nonunion. It may be difficult to achieve a stable fixation construct in infraisthmic fractures or infraisthmic nonunion, as the tightness of fit of the surface with the intramedullary nail is theoretically limited at the isthmic portion. Lack of a tight fit aggravates rotational instability [12], and exchange nailing may not provide sufficient stability for nonisthmal union of the femoral shaft. Although the associated use of interlocking screws may improve stability [13], rotational instability may originate from the shorter length of the distal fragment and the quality of bone around the interlocking screws. Distal interlocking screws play an important role in increasing axial and rotational stability, and their use is recommended to enhance distal fixation in infraisthmic nonunion [14]. We also applied this rationale, and achieved a successful outcome, in most cases (14 of 15) of infraisthmic nonunion fixed by three or more interlocking screws. When the selected nail did not allow distal fixation with multiple screws, Poller screws were chosen to achieve high stability. Inasmuch as we ultimately achieved successful healing in all 15 cases of infraisthmic nonunion, enhanced distal fixation may be valuable.

It is surprising that in all three cases of nonunion, the failures occurred at the isthmic level, which, according to a biomechanical viewpoint, should have been less likely to happen [12]. Although multiple factors were involved in these failures, the mechanical construct should be analyzed. In two cases of failure, the initial fracture was of the ipsilateral femoral neck and shaft, with associated injuries of the lower limb (tibia and calcaneus) on the same side. Although the level of nonunion was isthmic, the lack of immediate weight-bearing activity because of the multiple injuries likely created an environment less favorable for healing of nonunion after exchange nailing [8]. In the other case, the initial fracture was at the isthmic level and was accompanied by a medial bending wedge fragment (AO/OTA 32-B2). At the time of exchange nailing, the medial wedge was not united, which resulted in a larger diameter of the medullary canal. Although the isthmic level is thought to be biomechanically favorable for healing [15], increasing the nail diameter may not achieve sufficient tightness of fit when the fracture is comminuted or wedged. Even if nonunion is located at the isthmic portion, we suggest using different methods of osteosynthesis. In the cases of failure in our series, we performed exchange nailing with enhanced distal fixation (multiple distal screws), plate augmentation, or both to increase the stability at the nonunion site.

Although exchange nailing is successful in most cases 
of aseptic nonunion, oligotrophic or atrophic patterns of nonunion are known risk factors for failure after exchange nailing [16]. Exchange nailing alone is not recommended for the treatment of nonunion with a segmental defect or in cases in which the bone is foreshortened by more than $1 \mathrm{~cm}$ [17]. In this study, 21 of 22 cases (95.5\%) of hypertrophic nonunion had successful outcomes, whereas six of eight cases (75\%) of oligotrophic nonunion had successful outcomes. In the two failures of oligotrophic nonunion, we performed a revision procedure to increase the stability and thereby achieved healing. Therefore, we recommend the stronger method of osteosynthesis, rather than exchange nailing, for oligotrophic or atrophic nonunion.

Because enhanced distal fixation is recommended for infraisthmic nonunion of the femoral shaft, we also fixed multiple screws at the distal segment in 19 cases in which two screws were not expected to provide sufficient stability. In this study, the failure rate after the use of two distal screws (18.2\%) was higher than that after the use of multiple screws (5.3\%). Placing additional screws is not difficult in newer designs of femoral nails, and so we recommend using multiple interlocking screws for exchange nailing in most cases of femoral shaft nonunion.

The limitations of our study include its retrospective nature, the heterogeneity of the patients, and the small number of patients. However, this study has several strengths with regard to the inclusion criteria: we excluded cases of nonunion repaired with other methods, such as an external fixator or plate, as well as cases of infected nonunion. The occurrence of three failures in this study may be another limitation for determining the risk factors. A large-scale, multicenter prospective study may be needed, although femoral nonunion that necessitates exchange nailing is relatively uncommon.

\section{CONCLUSION}

In summary, exchange nailing is a highly reliable method of achieving successful healing of femoral shaft nonunion. Nonetheless, the authors still experienced some cases of nonunion after exchange nailing that needed more stability of the distal fragment. Therefore, refinements of the exchange nailing technique may be considered to reduce the likelihood of failure in risky cases that require further stability.

\section{REFERENCES}

1. Ricci WM, Bellabarba C, Evanoff B, Herscovici D, DiPasquale T, Sanders R. Retrograde versus antegrade nailing of femoral shaft fractures. J Orthop Trauma 2001;15:161-9.

2. Taitsman LA, Lynch JR, Agel J, Barei DP, Nork SE. Risk factors for femoral nonunion after femoral shaft fracture. J Trauma 2009;67:1389-92.

3. Oh JK, Bae JH, Oh CW, Biswal S, Hur CR. Treatment of femoral and tibial diaphyseal nonunions using reamed intramedullary nailing without bone graft. Injury 2008;39:952-9.

4. Park KC, Oh CW, Kim JW, Park KH, Oh JK, Park IH, et al. Minimally invasive plate augmentation in the treatment of longbone non-unions. Arch Orthop Trauma Surg 2017;137:1523-8.

5. Brinker MR, O'Connor DP. Exchange nailing of ununited fractures. J Bone Joint Surg Am 2007;89:177-88.

6. Pihlajamäki HK, Salminen ST, Böstman OM. The treatment of nonunions following intramedullary nailing of femoral shaft fractures. J Orthop Trauma 2002;16:394-402.

7. Yang KH, Kim JR, Park J. Nonisthmal femoral shaft nonunion as a risk factor for exchange nailing failure. J Trauma Acute Care Surg 2012;72:E60-4.

8. Shroeder JE, Mosheiff R, Khoury A, Liebergall M, Weil YA. The outcome of closed, intramedullary exchange nailing with reamed insertion in the treatment of femoral shaft nonunions. J Orthop Trauma 2009;23:653-7.

9. Weber BG, Brunner C. The treatment of nonunions without electrical stimulation. Clin Orthop Relat Res 1981;161:24-32.

10. Swanson EA, Garrard EC, Bernstein DT, O'Connor DP, Brinker MR. Results of a systematic approach to exchange nailing for the treatment of aseptic femoral nonunions. J Orthop Trauma 2015;29:21-7.

11. Banaszkiewicz PA, Sabboubeh A, McLeod I, Maffulli N. Femoral exchange nailing for aseptic non-union: not the end to all problems. Injury 2003;34:349-56.

12. Watanabe Y, Takenaka N, Kobayashi M, Matsushita T. Infra-isthmal fracture is a risk factor for nonunion after femoral nailing: a case-control study. J Orthop Sci 2013;18:76-80.

13. Gabarre S, Albareda J, Gracia L, Puértolas S, Ibarz E, Herrera A. 
Influence of screw combination and nail materials in the stability of anterograde reamed intramedullary nail in distal femoral fractures. Injury 2017;48(Suppl 6):S47-53.

14. Kim JW, Yoon YC, Oh CW, Han SB, Sim JA, Oh JK. Exchange nailing with enhanced distal fixation is effective for the treatment of infraisthmal femoral nonunions. Arch Orthop Trauma Surg 2018;138:27-34.

15. Millar MJ, Wilkinson A, Navarre P, Steiner J, Vohora A, Hardidge A, et al. Nail fit: does nail diameter to canal ratio predict the need for exchange nailing in the setting of aseptic, hypertrophic femoral nonunions? J Orthop Trauma 2018;32:245-250.

16. Tsang ST, Mills LA, Frantzias J, Baren JP, Keating JF, Simpson AH. Exchange nailing for nonunion of diaphyseal fractures of the tibia: our results and an analysis of the risk factors for failure. Bone Joint J 2016;98-B:534-41.

17. Gelalis ID, Politis AN, Arnaoutoglou CM, Korompilias AV, Pakos EE, Vekris MD, et al. Diagnostic and treatment modalities in nonunions of the femoral shaft: a review. Injury 2012;43:980-8. 